\title{
Anwendung der Gesprächsanalyse als Feedback-Instrument im Chat-Coaching
}

\author{
Oliver Winkler ${ }^{1}$ iD
}

Zusammenfassung Der Artikel zeigt den Nutzen der Gesprächsanalyse für das Kommunikationstraining von Coaches im Bereich Chat-Coaching auf. Im Fokus liegt die Gesprächssteuerung des Coaches, die als entscheidendes Kriterium einer gelungenen Gesprächsführung betrachtet wird. Gesprächssteuerung ist auf verschiedenen theoretisch unterscheidbaren Ebenen des Gesprächs beschreibbar. Basierend auf einem Korpus mit rund $30 \mathrm{~h}$ Chat-Beratung werden drei Ebenen der Gesprächssteuerung anhand von Beispielen erläutert: Auf der Ebene der Gesprächsorganisation wird das Phänomen der Überlappungen im Sprecherwechsel sowie die Frage nach effizienten und adressatengerechten Formen von Orientierungshandlungen untersucht. Auf der Ebene der Themenkonstitution wird die Relevanz von reformulierenden Handlungen für die wechselseitige Verstehenssicherung erläutert. Auf der Ebene der Handlungskonstitution wird gezeigt, wie durch Fragehandlungen von Seiten des Coaches Rollenzuweisungen erfolgen. Im Anschluss an die prozessorientierte Analyse der einzelnen Chat-Auszüge folgen jeweils Überlegungen dazu, wie die identifizierten trainierbaren Momente im Gespräch im Rahmen eines Kommunikationstrainings nutzbar gemacht werden können. Die Einzelanalysen zeigen, dass die Gesprächsanalyse einen prozessnahen Blick auf die Gesprächsführung erlaubt und auch Hinweise liefert, wie Gesprächsabläufe von Seiten der Coaches effizienter gestaltet werden können.

Oliver Winkler

wino@zhaw.ch

1 Forschungsbereich Sprachkompetenz und Wissensvermittlung, Departement Angewandte Linguistik, LCC Language Competence Centre, Zürcher Hochschule für Angewandte Wissenschaften, Theaterstrasse 17, Postfach, 8400 Winterthur, Schweiz
Schlüsselwörter Gesprächsanalyse · Chat · Coaching · Gesprächssteuerung $\cdot$ Kommunikationstraining

\section{Applying Conversation Analysis as a Feedback Tool in Chat Coaching}

Abstract This paper explores how conversation analysis can be used as a linguistic tool for coaching training, focusing on the computer-mediated form of chat coaching. Interaction management hereby represents a core element of successful coaching and can be analysed from distinct theoretical perspectives. Based on a corpus containing approximately thirty hours of chat conversation three levels of interaction management are defined and each level is elaborated by an example: The first level describes characteristics of turn-taking as well as aspects of global and local organisation in conversation, the second level concerns reformulation and its significance for mutual understanding and the third level relates to question techniques as a means of constructing roles. Each analysis of the examples is followed by considerations on how the identified trainable moments in talk can be turned into trainable skills and applied in a communication training setting. The findings indicate that the conversation analytic approach allows for process-oriented observations and provides an insight to how coaches interact efficiently in chat coaching.

Keywords Conversation analysis - Chat - Coaching . Interaction management $\cdot$ Communication training 


\section{Untersuchungsgegenstand Chat-Coaching}

Im Zuge der Digitalisierung unserer Gesellschaft, der heranwachsenden Online-Generation und der damit einhergehenden neuen Lebensweise, die zeitlich und räumlich flexibler wird, gewinnt der Bereich des E-Coachings zunehmend an Bedeutung (Ribbers und Waringa 2015). E-Coaching zeichnet sich dadurch aus, dass der Coachingprozess nicht mehr auf Kopräsenz zwischen Coach und Klient basiert, sondern computervermittelt und damit unabhängig von geographischen Beschränkungen stattfinden kann. Während Beratung auf Distanz bis vor kurzem nur per Telefon möglich war, wird immer häufiger auch auf andere Kanäle wie Video, E-Mail und Chat zurückgegriffen (Ribbers und Waringa 2015). Diese verhältnismäßig neuen Kanäle stellen aufgrund ihrer medialen Beschaffenheit ganz bestimmte Anforderungen sowohl an die Klienten als auch die Coaches. Gerade der Chat bleibt hierbei eine besondere Herausforderung, weil er als Kommunikationsform weder dem in professionellen Kontexten etablierten E-Mail-Text noch dem prototypisch mündlichen Gespräch entspricht, sondern sich auf einem Kontinuum zwischen Merkmalen konzeptioneller Schriftlichkeit und Mündlichkeit bewegt (Koch und Oesterreicher 1985) und somit im Beratungskontext für viele eine neue Kommunikationsform darstellt.

Die folgende Studie soll zeigen, dass die linguistische Disziplin der Gesprächsanalyse ein nützliches Instrument bietet, um Elemente der Gesprächssteuerung (Gesprächsorganisation, Themen- und Handlungskonstitution) im ChatCoachingprozess zu identifizieren und daraus Hinweise abzuleiten, wo potenzielle Herausforderungen und Verbesserungsmöglichkeiten im Gesprächsverhalten von Coaches liegen. Materialbasis ist ein Chat-Korpus mit knapp $30 \mathrm{~h}$ Chat-Beratungen. Die Coaches sind Studierende des Masterstudiengangs Angewandte Psychologie an der Zürcher Hochschule für Angewandte Wissenschaften ZHAW und führen die Coachings im Rahmen des Pflichtmoduls „Distanzberatung" durch. Die Coachings finden auf der Plattform $\mathrm{CAI}^{\circledR}$ World statt. ${ }^{1}$ Die Studierenden werden damit beauftragt, eine ihnen unbekannte Person mit einem echten Anliegen zu beraten.

\section{Gesprächsanalytische Kommunikationstrainings}

Vom Gegenstand und der Methode her gliedert sich die Studie ein in den Bereich der angewandten Gesprächsana-

\footnotetext{
${ }^{1}$ Diese Plattform ermöglicht die Kombination von Audio-, audiovisueller sowie Chat-Kommunikation. Die vorliegenden Gespräche sind jedoch reine Chat-Mitschnitte.
}

lyse bzw. der Applied Conversation Analysis. Antaki (2011, S. 1) bestimmt deren Gegenstand dahingehend, ,that the application of Conversation Analysis (CA) to the talk of an institution like the school or the medical clinic can shed light on its workings; and that the CA researcher can suggest improvements in the service that such an institution provides“. Mit Blick auf die deutschsprachige angewandte Gesprächsanalyse haben sich etwa im klinischen Bereich der Arzt-Patienten-Kommunikation (vgl. bspw. Lalouschek 2002, 2004, 2005; Nowak 2010) oder im Bereich der schulischen Kommunikation (vgl. bspw. BeckerMrotzek und Brünner 2006; Becker-Mrotzek 2009; Harren 2015; Meer 2007; Spiegel 2006) domänenspezifische Forschungsbereiche der angewandten Gesprächsanalyse entwickelt. Obwohl auch gesprächsanalytisch fundierte Kommunikationstrainings in diversen beruflichen Kontexten bereits erprobt wurden (Meer und Spiegel 2009), machen diese im Vergleich mit kommunikationspsychologischen Ansätzen wie etwa jenem von Schulz von Thun (2014) immer noch einen marginalen Anteil im kommunikativen Weiterbildungsmarkt aus (Hartung 2004). Auch Antaki (2011, S. 9) stellt für den englischsprachigen Raum fest, die Gesprächsanalyse sei außerhalb des eigenen Fachbereichs ,,not yet in the phone book". Allerdings wurden in den letzten Jahren gerade im englischsprachigen Kontext der CA gesprächsanalytische Trainingskonzepte entwickelt, die auch im beruflichen Weiterbildungsmarkt Anklang finden und kommerziell verbreitet werden. Erfolg zu verzeichnen hat etwa der von Elizabeth Stokoe und ihrem Team entwickelte CARM-Ansatz (Conversational Analytic Role-play Method) (Stokoe 2011, 2013, 2014), welcher der „Interventionist applied CA“ (Antaki 2011, S. 8) zuzuordnen ist.

Ansätze wie jener von Stokoe gehen von einer ,,coproduction of interventions between academics and practitioners“ aus, wobei die berufstätige Person als „the primary owner of expertise" (Hepburn et al. 2014, S. 240) angesehen wird. Dem praktischen Erfahrungswissen der berufstätigen Person steht das analytische Wissen des Gesprächsanalytikers gegenüber, der Konzepte und Kategorien vermitteln kann, welche eine prozessnahe und detaillierte Beschreibung jeweiliger interaktionaler Praktiken erlauben. Zentral ist dabei, dass es sich um ein evidenzbasiertes Training handelt, das auf empirischem Gesprächsmaterial gründet, welches aus der Organisation oder Institution stammt, in der die zu trainierende Person arbeitet.

Entstanden ist CARM aus einem gesprächsanalytischen Forschungsprojekt über Streitigkeiten zwischen Nachbarn, in dem ein umfangreiches Korpus (120h) mit Mediationsgesprächen, Polizeiverhören sowie Erstkontaktaufnahmen zwischen Betroffenen und kommunalen Mediationsdiensten untersucht wurde. Besonders die Ergebnisse aus den Analysen von Erstkontaktaufnahmen zwischen Klient und Mediationsdienst stießen aus Sicht der Mediationsdienste 
auf Interesse und wurden dann in Form von Workshops mit der involvierten Zielgruppe (Mediatoren) als Grundlage für Kommunikationstrainings produktiv genutzt (Stokoe 2011).

Ausgangspunkt eines CARM-Workshops ist das Sammeln und Transkribieren von Gesprächsmaterial. ${ }^{2}$ Darauf basierend werden vorgängig Stellen identifiziert, die vor dem Hintergrund des institutionellen Kontextes entweder Kommunikationsprobleme oder geglückte Lösungen kommunikativer Aufgaben darstellen. Kern des Workshops bildet dann die detaillierte Auseinandersetzung und Simulation von Gesprächen auf der Grundlage der anonymisierten Gesprächstranskripte (Stokoe 2011). Der im Namen CARM enthaltene Begriff „Role-play“ bedeutet, dass die Teilnehmenden auf der Basis des Gesprächstranskripts das Gespräch durchspielen, indem sie Schritt für Schritt Turns $^{3}$ analysieren und darauffolgende Reaktionen formulieren und diskutieren:

The transcript is presented line-by-line, synchronised with the audio file. This means that workshop participants "live through" the call as it happens - they do not receive transcripts ahead of hearing the extract and do not know how the conversation unfolds beyond the lines I play to them. (Stokoe 2011, S. 126)

Das Ziel eines solchen gesprächsanalytischen Kommunikationstrainings ist, dass die Teilnehmenden ein schärferes Bewusstsein für ihre eigenen Praktiken entwickeln, für gewisse Probleme und Schwierigkeiten im Gesprächsprozess - Stokoe (2014, S. 258) spricht hier von ,racetracks“ - sensibilisiert werden und die Möglichkeit erhalten, das eigene Handlungsspektrum zu erweitern.

Die CARM-Methode wurde auf der Grundlage von audio- und audiovisuellen Gesprächen entwickelt, wobei Stokoe (2014, S. 262) auf die Ausweitbarkeit auf schriftliche Texte hinweist. Dennoch bedarf es im Folgenden einer näheren Erörterung darüber, wo die Differenzen und Parallelen zwischen den zwei Diskursformen des mündlichen Face-to-Face-Gesprächs und des schriftlichen Online-Chats liegen und weshalb es naheliegt, gesprächsanalytische Kategorien auf den Gegenstand des Online-Chats anzuwenden.

\section{Chat-Coaching als Gesprächsform}

Dass man das gesprächsanalytische Instrumentarium für die Untersuchung medial schriftlicher Dialogformen nutzbar machen kann, hat bereits die historische Dialogforschung,

\footnotetext{
${ }^{2}$ Stokoe macht keine genauen Angaben bzgl. des quantitativen Umfangs der verwendeten Gesprächsdaten.

${ }^{3}$ Ein Turn kann nach Goffman (1974, S. 201) als ,,alles, was ein Individuum tut und sagt, während es jeweils an der Reihe ist", definiert werden.
}

die sich naturgemäß auf schriftliche Quellen beziehen muss, erkannt und gezeigt (Henne 1980; Hess-Lüttich 1981; Kilian 2005). Hess-Lüttich und Wilde (2003, S. 166) bringen den zentralen Wesenszug von Chat-Dialogen auf den Punkt, wenn sie festhalten: „Chat-Kommunikation gilt als Hybridmedium im Interferenzfeld zwischen geschriebener und gesprochener Sprache, auch im Bewusstsein der Teilnehmer: sie schreiben ihren Beitrag als Rede-Beitrag in einem medial vermittelten Gespräch." Die Unterschiede sind freilich nicht nur medialer Art, vielmehr lassen sich eine Reihe von Merkmalen feststellen, welche den Chat und das Face-toFace-Gespräch theoretisch als zwei verschiedene Diskursformen bestimmen lassen. Nebst der räumlichen Trennung und der damit einhergehenden fehlenden Kopräsenz der Interaktanten spielt vor allem die maschinelle Vermittlungsinstanz des Servers eine wesentliche Rolle, da dieser keine synchrone, sondern lediglich „quasi-synchrone“ (Dürscheid und Brommer 2009, S. 6) Interaktion zulässt. Während Produktion und Rezeption von Gesprächsbeiträgen im Face-to-Face-Gespräch synchron ablaufen, finden diese im Chat-Dialog stets „konsekutiv“ (Beisswenger 2007, S. 49) statt. Weder kann der Rezipient einer Nachricht den Entstehungsprozess einer Nachricht und den genauen Zeitpunkt des Versendens nachvollziehen, noch weiß der Produzent der Nachricht, wann genau seine Nachricht rezipiert wird. Somit ist nicht garantiert, dass die wechselseitigen Redebeiträge gleichzeitig wahrgenommen werden, weshalb Beisswenger (2007, S. 50) von einer unterschiedlichen „Ereigniswahrnehmung" spricht. Diese maschinellen Bedingungen wirken sich gerade auf den Gesprächsbereich der psychologischen Beratung aus. Während im Face-to-Face-Coaching-Gespräch unmittelbare und spontane, vielleicht auch unbewusste Reaktionen non- und paraverbaler Art geradezu konstitutiv für den Gesprächsverlauf sind, bleiben diese Zeichen im Chat-Dialog verborgen, solange sie nicht verbal oder in Form von z. B. Emoticons zum Ausdruck gebracht werden. Nebst diesen durch das Medium der Schrift reduzierten Möglichkeiten an Zeichenkanälen unterscheiden sich auch Organisation und Geschwindigkeit des Sprecherwechsels von natürlichen Gesprächen. Studien von Beisswenger (2007) und Thaler (2003) weisen nach, dass sich das System des Turn-taking (Sacks et al. 1974) aufgrund des konsekutiven Ablaufs und der damit verbundenen Entkoppelung der Beiträge anders gestaltet als in mündlichen Gesprächen. So ist beispielsweise eine Unterbrechung im herkömmlichen Sinn einer Turn-Übernahme nicht möglich, durchaus jedoch in Form von abrupten Themenwechseln oder durch Flooding (Einspeisen großer Textmengen), wie es in Mehrparteien-Chats der Fall sein kann (Thaler 2003). Daran zeigt sich, dass bei der Verwendung gesprächsanalytischer Kategorien die genannten medial bedingten Eigenschaften berücksichtigt werden müssen (Hess-Lüttich und Wilde 2003). 
Einmal abgesehen von den oben genannten Differenzen weist das Chat-Gespräch auch deutliche Parallelen zum mündlichen Gespräch auf. Das zeigt sich etwa darin, dass sich die von Deppermann (2008, S. 8) genannten fünf Merkmale von Gesprächen auf den Gegenstand von Chat-Gesprächen übertragen lassen: Chats sind konstitutiv, d.h. die Chat-Ereignisse ,werden von den Gesprächsteilnehmern aktiv hergestellt", sie verlaufen prozessartig $\mathrm{ab}$, sind somit ,zeitliche Gebilde, die durch eine Abfolge von Aktivitäten entstehen". Chats sind interaktiv, da sie aus ,wechselseitig aufeinander bezogenen Beiträgen“ bestehen, ${ }^{4}$ und sie unterliegen dem Kriterium der Methodizität, indem die Gesprächsteilnehmenden ,für andere erkennbare und verständliche Methoden“ benutzen, um sich zu verständigen. Chats tragen ebenfalls das Merkmal der Pragmatizität, indem die Teilnehmenden ,gemeinsame und individuelle Zwecke [verfolgen]" sowie „Probleme und Aufgaben [bearbeiten]". Gerade anhand der beiden wesentlichen Merkmale der Prozessualität und Interaktivität zeigt sich, dass der Chat seinem Wesenszug nach dem prototypischen mündlichen Gespräch nähersteht als dem prototypisch schriftlichen Text, womit auch die Nutzbarmachung gesprächsanalytischer Kategorien anstelle etwa von textlinguistischen sinnvoll erscheint.

\section{Gesprächssteuerung im Chat-Coaching}

Coachinggespräche gehören in den Bereich institutioneller Kommunikation, wodurch bestimmte Rahmenbedingungen (örtliche und zeitliche Dimension, Grad der Öffentlichkeit, Bekanntheitsgrad der Gesprächspartner, Status und Rollen) vorgängig festgelegt sind. Mit der Kommunikation zwischen Ratsuchendem und Ratgebendem treffen auch unterschiedliche Perspektiven, Referenzsysteme und Wissenshintergründe sowie rollenspezifische interaktive Aufgaben aufeinander (Nothdurft et al. 1994). Zu den zentralen Aufgaben des Coaches gehört es, das Gespräch im vorgegebenen zeitlichen und inhaltlichen Rahmen zu organisieren, zu strukturieren und zu steuern. Wohlwissend, dass eine erfolgreiche Organisation und Steuerung des Gesprächs nicht nur vom Coach, sondern in erheblichem Maße auch vom Klienten abhängt, liegt der Hauptfokus der nachfolgenden Analysen auf den gesprächssteuernden Aktivitäten des Coaches. Ziel ist, auf der Basis des vorhandenen Materials trainierbare Momente zu identifizieren und zu zeigen, wo und wie gesprächsanalytische Beobachtungen und Ergebnisse in der Aus- und Weiterbildung von Coaches einfließen können.

\footnotetext{
${ }^{4}$ Wobei die Organisationsprinzipien insbesondere bei MehrparteienChats eben anders gestaltet sind als im natürlichen Gespräch (Thaler 2003).
}

Unter gesprächssteuernden Aktivitäten können mit Tiittula (2001, S. 1361) solche verstanden werden, ,mit denen die Interagierenden auf den Verlauf des Gesprächs lenkend einwirken, indem sie z. B. versuchen zu regeln, wer das Rederecht erhält, worüber gesprochen wird und welche Handlungen die Gesprächspartner durchzuführen haben." Gesprächssteuerung findet sowohl auf der verbalen als auch der non- und paraverbalen Ebene des Gesprächs statt. Laut Tiittula (2001, S. 1364) kann die verbale Gesprächssteuerung auf mindestens drei zentralen Ebenen analysiert werden: ,(1) auf der Ebene der Gesprächsorganisation (hierzu gehören u. a. Verteilung der Redegelegenheiten und Sprecherwechsel), (2) auf der Ebene der Handlungskonstitution [durch initiierende Handlungen implizierte Erwartungen und Folgehandlungen] und (3) auf der Sachverhaltsebene (Darstellung der Sachverhalte und thematische Entfaltung).“Als weitere Ebenen nennt sie ebenfalls die Beziehungskonstitution sowie die Interaktionsmodalität (z. B. Humor, Spiel).

Wenn Lalouschek (2004, S. 141) für die Arzt-Patienten-Interaktion aus Sicht des Praktizierenden ein gewisses Dilemma zwischen dem Anspruch an Patientenorientiertheit und dem Anspruch an Effizienz feststellt, gilt das auch für die vorliegenden Chat-Coaching-Gespräche. Auch hier steht der Anspruch an Klienten-Orientierung und Beziehungsgestaltung dem Anspruch an effizienter Gesprächsführung im Sinne einer inhaltlichen, zeitlichen und technischen Begrenzung gegenüber (Ribbers und Waringa 2015; Seidlitz und Theiss 2008). Gerade die weiter oben thematisierten Implikationen computervermittelter Kommunikation stellen neue Ansprüche an die Gesprächsführung. Nicht nur aufgrund der bereits genannten Quasi-Synchronizität, sondern auch aufgrund der unterschiedlichen technischen Fähigkeiten und Erfahrungen der Klienten im computervermittelten Schreiben und Kommunizieren, auf die Coaches angemessen reagieren müssen.

In Anlehnung an den CARM-Ansatz werden aus dem vorliegenden Material im Folgenden solche Gesprächsausschnitte gewählt, die im Training als Veranschaulichungsbeispiel zur Sensibilisierung und Bewusstmachung von potenziellen Hindernissen/Problemen - Stokoe (2014, S. 256) spricht von „Roadblocks“ - dienen.

\section{Identifizierung von trainierbaren Momenten im Gespräch}

\subsection{Materialauswahl und Verfahrensweise}

Materialgrundlage bildet ein Korpus mit insgesamt neun Coachings. Die Coachings ziehen sich über zwei bis vier Sitzungen, die meistens 45-60 min dauern. Sämtliche invol- 
vierte Personen sind Frauen. Die Coaching-Paare wurden per Los gebildet. ${ }^{5}$

Um Möglichkeiten der Nutzbarmachung gesprächsanalytischer Beobachtungen exemplarisch zu zeigen, sollen im Folgenden vier verschiedene trainierbare Momente erläutert werden, die jeweils einer der genannten drei Ebenen der Gesprächssteuerung (Gesprächsorganisation, Handlungskonstitution und Sachverhaltsdarstellung) zuzuordnen sind. Gleichzeitig ist die Auswahl der Beispiele in den spezifischen Merkmalen des Gesprächstyps Coachinggespräch und der Kommunikationsform des Chats begründet.

Als Beispiel für die Gesprächsorganisation werden der Sprecherwechsel sowie Formen von Orientierungshandlungen beleuchtet. Während Orientierungshandlungen ein zentrales Mittel der Prozesssteuerung und -strukturierung sind, ist der Sprecherwechsel ein Basisprinzip, das jedoch im Chat aufgrund der maschinellen Vermittlungsinstanz andere Gesetzmäßigkeiten als im Face-to-Face-Gespräch zeigt und ein potenzielles Hindernis für eine effiziente Gesprächsführung darstellt. In Hinblick auf die Sachverhaltsebene wird das Phänomen reformulierender Handlungen thematisiert, während auf der Ebene der Handlungskonstitution FrageAktivitäten behandelt werden. Frage-Aktivitäten und Reformulierungen repräsentieren beide gesprächstypspezifische Phänomene, die jedoch durch den Chat ebenfalls eine kommunikationsformspezifische Prägung erfahren.

In Anlehnung an das Vorgehen bei Lalouschek (2004) werden die Gesprächsausschnitte in einem ersten Schritt analysiert und in einem zweiten Schritt hinsichtlich der Möglichkeit einer konkreten Bearbeitung im Kommunikationstraining reflektiert.

Bei den Gesprächen handelt es sich um Chat-Mitschnitte, die direkt aus der Plattform CAI ${ }^{\circledR}$ World exportiert wurden. Die Gesprächsausschnitte stammen aus vier verschiedenen Coaching-Sitzungen. ${ }^{6}$ Die Abkürzungen $\mathrm{C}$ bzw. K stehen für Coachin bzw. Klientin.

\subsection{Sprecherwechsel}

Ein zentraler Aspekt der Gesprächsorganisation sind die Ordnungsprinzipien des Sprecherwechsels, d. h. die Verteilung und Zuweisung des Rederechts in einem Gespräch. Nach Sacks et al. (1974) können drei Arten des Sprecherwechsels unterschieden werden: 1. Ein aktueller Sprecher wählt den nächsten. 2. Ein nächster Sprecher wählt sich selbst aus. 3. Der aktuelle Sprecher wählt sich selbst, wenn niemand anderes das Wort ergreift. Während diese Systematik für Face-to-Face- und Telefongespräche gilt, weisen

\footnotetext{
5 Eine detailliertere Beschreibung der Stichprobe findet sich in Von Arx und Rüsch (2016, 14 ff.).

${ }^{6}$ Bei den Ausschnitten in Abschn. 5.2 und 5.3.1 handelt es sich jedoch um die gleiche Dyade.
}

Studien zur Chat-Kommunikation aufgrund der technischen Bedingungen andere Gesetzmäßigkeiten des Sprecherwechsels nach. Herring (2001, S. 618) verweist auf zwei Hindernisse, die sich bei der Gesprächssteuerung auf der Ebene des Sprecherwechsels im Chat ergeben: ,(1) disrupted turn adjacencey caused by the fact that messages are posted in the order received by the system, without regard for what they are responding to, and (2) lack of simultaneous feedback caused by reduced audiovisual cues." Beides führt im Chat-Gespräch häufig zu thematischen Überlappungen, die dann in Form von Reparaturhandlungen aufgelöst werden müssen (vgl. bspw. Hess-Lüttich und Wilde 2003; Thaler 2003). Auch im vorliegenden Material treten Turn-Überlappungen mit anschließenden Reparaturhandlungen auf. Der folgende Gesprächsausschnitt zeigt dies exemplarisch:

C7: 24.11. 12:12 - Ich hab mir unsere letzte Sitzung nochmal angeschaut und mir ein paar Dinge überlegt C8: 24.11. 12:12 - Konnten sie sich noch Gedanken bzgl. Stärkung des Kindes/der Fee machen?

K4: 24.11. 12:13 - Konkret nicht, aber ich habe so eine typische Situation vorletzte Woche erlebt C9: 24.11. 12:13 - wollen sie mir davon erzählen? K5: 24.11. 12:13 - ja, oder haben sie was anderes geplant?

K6: 24.11. 12:13 - vielleicht können sie mir anhand von dieser situation auf das nächste mal tipps geben? C10: 24.11. 12:13 - nein, ich werde ihnen danach gerne sagen, was ich mir überlegt habe K7: 24.11. 12:14 - nur so ein gedanke ...

K8: 24.11. 12:14 - ok

C11: 24.11. 12:14 - genau das ist doch gut!

Analyse und Bearbeitung Der Ausschnitt beginnt geordnet, indem auf den initiierenden Turn durch die Coachin (Frage) in Form von zwei Chunks (C7, C8) ein respondierender Turn (Antwort) der Klientin (K4) folgt. Darauf folgt ein weiterer initiierender Turn der Coachin (C9), auf den die Klientin mit einem reaktivierenden Turn ${ }^{7}$ reagiert, der in Form von zwei Chunks erfolgt (K5, K6). Dies führt nun zu einer nachfolgenden Überlappung der Gesprächsbeiträge: C10 nimmt auf die Frage in K5 Bezug, wobei K6 zunächst unbeantwortet bleibt. K7 wiederum bezieht sich nicht auf $\mathrm{C} 10$, sondern knüpft an $\mathrm{K} 6$ an. Erst der darauffolgende Chunk K8 „ok“ respondiert auf C10. Die entstandene Verwicklung wird dann in C11, ,aufgelöst“, indem die Coachin auf den Vorschlag in K6 eingeht.

In den neun untersuchten Coachings zeigt sich, dass lediglich in zwei Chat-Ereignissen die Organisation des Spre-

\footnotetext{
7 Reaktivierende Turns sind Turns, die sowohl eine respondierende als auch eine initiierende Komponente enthalten (vgl. Brinker und Sager 2010).
} 
cherwechsels zu Beginn der Sitzung von Seiten der Coachin explizit thematisiert wird. Im Workshop kann anhand von solchen Beispielen das Problem der maschinellen Vermittlung von Turns und der diskrepanten Ereigniswahrnehmung gezeigt und problematisiert werden. In Hinblick auf das Kriterium der Effizienz kann diskutiert werden, inwiefern eine vorgängige Verabredung von Konventionen der Regelung des Turnwechsels im Kontext der Beratungsgespräche wünschenswert und realisierbar ist. Ebenfalls können Möglichkeiten der Regulierung des Rederechts, wie z.B. Formen der „Fortsetzungsmarkierung“ (Storrer 2001, S. 14), in Hinblick auf ihren situativ angemessenen Einsatz besprochen werden.

\subsection{Orientierungshandlungen}

\subsubsection{Globale Orientierungshandlungen}

Globale Orientierungshandlungen sind Handlungen, die über die Struktur und den Verlauf des Gesprächs Aufschluss geben und die sich naturgemäß dadurch vor allem am Gesprächsbeginn bzw. -ende befinden. Es handelt sich somit um Phänomene der makrostrukturellen Gesprächsorganisation. Globale Orientierungshandlungen zu Beginn des Gesprächs haben die Aufgabe, dass sich die Interaktanten wechselseitig über Gesprächszweck, -ziel und -verlauf orientieren (Lalouschek 2004), wie das folgende Beispiel exemplarisch zeigt:

C1: 01.11. 13:19 - Liebe Frau C schön sind sie da!

C2: 01.11. 13:20 - Herzlich Willkommen zu unserer

1. Sitzung

C3: 01.11. 13:20 - Haben Sie den Fragebogen, den ich Ihnen per Mail zuschickte noch gesehen?

K1: 01.11. 13:20 - Liebe Frau G, freut mich ebenfalls!

K2: 01.11. 13:20 - Ja, habe ihn bereit ausgefüllt.

C4: 01.11. 13:20 - SUper!!

C5: 01.11. 13:20 - Herzlichen Dank!

K3: 01.11. 13:21 - Gern geschehen

C6: 01.11. 13:21 - Vielleicht klären wir zuerst noch ein paar formelle Dinge bevor es mit der Beratung losgeht

K4: 01.11. 13:21 - Ja, das wäre gut

C7: 01.11. 13:21 - Wie sie wissen haben wir ca. 3-4 Sitzungen à ungefähr 45 Minuten Zeit

C8: 01.11. 13:22 - Falls Sie jedoch nach kürzerer Zeit das Gefühl haben ,,jetzt isch gnueg“" schreiben Sies mir, dann können wir kürzere Sitzungen und dafür mehr machen

K5: 01.11. 13:23 - Ja, ist gut

C9: 01.11. 13:23 - Auch wenn Sie eine Anmerkung oder Frage von mir nicht verstehen einfach nachfragen! Ich werde das auch tun
C10: 01.11. 13:23 - Etzt habe ich noch eine Formfrage

C11: 01.11. 13:24 - Wie ist es Ihnen lieber sind sie wohl mit Siezen oder ist Ihnen Duzen lieber?

C12: 01.11. 13:24 - möchten Sie lieber Mundart schreiben?

K6: 01.11. 13:24 - Siezen wäre gut und lieber hochdeutsch

C13: 01.11. 13:24 - bezüglich dem Mundartschreiben dürfen Sies machen wies gerade kommt

C14: 01.11. 13:24 - ok

C15: 01.11. 13:24 - das ist gut!

C16: 01.11. 13:25 - Ist es für Sie ok wenn wir wegen dem nächsten Termin am Ende der Sitzung schauen?

K7: 01.11. 13:25 - Ja

C17: 01.11. 13:26 - Super, haben Sie noch Fragen zu den Rahmenbedingungen oder zum Ablauf?

K8: 01.11. 13:26 - Ich gehe davon aus, dass Sie nun ein paar Fragen stellen werden und wir so irgendwie auf mein Thema zu sprechen kommen?

C18: 01.11. 13:26 - Noch eine Anmerkung von mir:

Sitzungen würde ich so ca. auf einmal wöchentlich verteilen, wenn das ok ist

K9: 01.11. 13:27 - Ja, ist ok

Analyse und Bearbeitung Das Gespräch beginnt mit einer Kontaktaufnahme (C1-K3), in der neben beziehungsaufbauenden Aktivitäten des freundlichen Grüßens von Seiten der Coachin abgesichert wird, dass die notwendigen Vorbereitungen (Ausfüllen des Formulars) erledigt wurden (C3), was durch die Klientin bestätigt wird (K2), worauf eine Dankessequenz (C4-K3) folgt. In C6 kündigt die Coachin an, im Vorfeld der Beratung ein paar formelle Dinge besprechen zu wollen, was von der Klientin bestätigt wird (K4). Die Coachin weist dann auf die vorvereinbarten zeitlichen Rahmenbedingungen hin (C7), die sie dann jedoch relativiert, indem sie der Klientin eine Alternative eröffnet (C8). Diese reagiert auch hier lediglich bestätigend (K5), worauf die Coachin den Fokus auf die Interaktionsmodalitäten verschiebt mit dem Hinweis an die Klientin, bei Verständnisschwierigkeiten einfach nachzufragen (C9). Innerhalb des gleichen Turns stellt sie dann die Frage nach der bevorzugten Anrede (Siezen, Duzen) und Sprache (Mundart, Hochdeutsch), worauf die Klientin ihre Präferenzen nennt (K6). Wohl aufgrund einer Überlappung folgt darauf in $\mathrm{C} 13$ nochmals ein Kommentar zum Thema „Mundartschreiben“, was dann jedoch in C15 repariert wird. Im gleichen Turn folgt ein Vorschlag bzgl. der Vereinbarung eines nächsten Termins (C16), der von der Klientin ebenfalls bestätigt wird (K7). Die Coachin ratifiziert das bisher Gesagte (Super) und lässt mit ihrer Frage der Klientin die Gelegenheit, weitere Fragen zu stellen (C17). Diese 
reagiert darauf in Form einer Vermutung (K8) bezüglich des weiteren Verlaufs, worauf die Coachin im Folgenden nicht eingeht, sondern in Form eines Nachtrags den thematischen Fokus zurück auf die terminliche Organisation der Sitzungen steuert (C18). Auch dieser Vorschlag wird von der Klientin bestätigt (K9).

Das oben thematisierte Spannungsfeld von Klientenorientiertheit einerseits und Effizienz andererseits wird anhand dieses Gesprächsausschnitts sichtbar. Einerseits zeigt sich von Seiten der Coachin das Bemühen um eine nicht-hierarchische Interaktion, bei der die Klientin die Möglichkeit erhält, die Gestaltung des Gesprächs zu beeinflussen und an die eigenen Bedürfnisse anzupassen. Andererseits wird anhand des sequentiellen Ablaufs erkennbar, dass die initiierten Orientierungshandlungen der Coachin schlecht strukturiert sind. Das zeigt sich besonders in Turn C18, wo die Coaching nicht auf die geäusserte Annahme der Klientin zum konkreten Gesprächsverlauf eingeht, sondern in abrupter Weise den Themenfokus zurück auf organisatorische Fragen (Sitzungsrhythmus) verschiebt. Da man sich bereits in der Sequenz C16-K7 darüber geeinigt hat, den nächsten Sitzungstermin am Ende der Sitzung zu besprechen, scheint die Information in C18 nicht gut platziert und führt vor allem dazu, dass die zentrale Frage der Klientin zum konkreten Gesprächsablauf unbeantwortet bleibt.

Dieser Gesprächsausschnitt kann in einer Bearbeitung im Kommunikationstraining als Ausgangspunkt fungieren für eine Diskussion der Frage, welche inhaltlich-thematischen Elemente Gegenstand dieser Orientierungshandlungen sein sollten - so werden hier etwa nur Fragen des formalen Ablaufs, aber keine inhaltlichen Aspekte und Zielvorstellungen besprochen. Andererseits kann anhand des Gesprächsausschnitts diskutiert werden, wie die gemeinsame Orientierung effizienter strukturiert und gesteuert werden kann.

\subsubsection{Lokale Orientierungshandlungen}

Im Gegensatz zu den globalen Orientierungshandlungen sind die lokalen Orientierungshandlungen zuständig für die Binnenstrukturierung von Gesprächen, indem sie den „Wechsel von einem Gesprächsabschnitt zu einem anderen“ (Lalouschek 2004, S. 152) regulieren:

C9: 03.11. 19:47 Verstehe ich Sie richtig, Sie tragen sich momentan mit der Überlegung ev. eine neue Ausbildung zu absolvieren und würden sich wünschen, dass wir gemeinsam schauen könnten welche Stärken Sie mitbringen?

K9: 03.11. 19:54 Sicherlich würden mich, die Stärken, die ich mitbringe interessieren, wenn ich diese auch für eine evtl. Ausbildung nutzen könnte, wäre es ja schön.
C10: 03.11. 19:56 Das stimmt. Aber es geht Ihnen mehr um das noch mögliche Potenzial in Ihnen? K10: 03.11. 20:01 Stimmt. Es geht mir vorwiegend um das noch mögliche Potenzial in mir und auch darum, welche Ausbildung oder Weiterbildung im jetzigen Arbeitsgebiet für mich in Frage kämen.

C11: 03.11. 20:06 Ok. Eine Potenzialabklärung in berufsberaterischen Sinn können wir leider innerhalb dieser drei Sitzungen nicht miteinander machen. Ich könnten Ihnen anbieten, das wir Perspektiven entwickeln, wie sie Antworten auf diese Fragen bekommen könnten. Wäre das in Ordnung für Sie?

K11: 03.11. 20:12 Ok. Perspektiven entwickeln klingt spannend. Geht in Ordnung.

C12: 03.11. 20:13 Sie haben oben geschrieben, dass Sie sich fragen ob sich eine neue Ausbildung noch lohnen würde. Woran würden Sie merken, dass sie sich gelohnt hat?

Analyse und Bearbeitung In den Turns C9-K10 verständigen sich Coachin und Klientin über das Anliegen. Dieses wird im Anschluss von der Coachin in C11 zunächst zurückgewiesen (Abklärung in berufsberaterischem Sinn), worauf sie jedoch der Klientin eine Alternative anbietet (C11). Der Ausdruck Perspektiven sowie die Formulierung diese Fragen - Verwendung des Determinativpronomens sind hinsichtlich ihres Referenzbezugs unklar. Zwar nimmt die Klientin in K11 diesen Vorschlag an $(O k$.$) , die direk-$ te Übernahme des Wortlauts (Perspektiven entwickeln), die Bewertung (klingt spannend) und die nachgeschobene wiederholte Bestätigung (geht in Ordnung) deuten zumindest an, dass sie sich unter dem Vorschlag der Coachin noch wenig Konkretes vorstellen kann. Dennoch vollzieht die Coachin in $\mathrm{C} 12$ einen Wechsel in die Klärungsphase in Form eines Rückbezugs auf eine Aussage der Klientin und einer damit verknüpften hypothetischen Frage.

Aus der Sicht der Beratungsexpertin bilden Fragen wie in C12 ein gängiges Mittel, um mit den Klienten Lösungsvisionen zu erarbeiten und die ,innere Landkarte“ (Seidlitz und Theiss 2008, S. 49) zu erweitern. Daher ist die Kohärenz zwischen dem Vorschlag in C11 (Perspektiven entwickeln) und der anschließenden Frage in C12 vorhanden. Aus Sicht der Klientin ist jedoch fraglich, ob dieser Zusammenhang verständlich wird und hier eine gemeinsame Orientierung in Bezug auf den Wechsel von einem Gesprächsabschnitt zum anderen vorliegt. Im Training können Möglichkeiten diskutiert und konkrete Formulierungsvarianten geübt werden, wie zwischen einzelnen Abschnitten Kohärenz deutlicher hergestellt und somit gemeinsame Orientierung gewährleistet werden kann. 


\subsection{Reformulierungshandlungen}

Reformulierungshandlungen treten in Beratungsgesprächen häufig auf. Sie sind ein Mittel zur interaktiven Konstitution einer gemeinsamen Verstehensbasis und der thematischen Gesprächssteuerung. Mit einer reformulierenden Handlung lässt sich u. a. ein bestimmter Fokus im Gespräch setzen oder auch eine gezielte Themenverschiebung, mitunter auch mit dem Ziel des Ausweichens (Tiittula 2001), vollziehen. Bührig (1996) hat gezeigt, dass Reformulierungshandlungen sowohl von Seiten des Ratsuchenden als auch von Seiten des Ratgebenden eingesetzt werden. Bei der Analyse des folgenden Beispiels liegt der Fokus auf der Coachin:

C20: 04.11. 16:26 - Präzisieren Sie dies bitte noch etwas. Was meinen Sie mit eine Veränderung Ihres Verhaltens und Ihrer Denkweise.

K19: 04.11. 16:27 - Ich gehe davon aus, dass ein grosser Anteil hier drin meine Sicht der Dinge ist.

K20: 04.11. 16:28 - Ich frage mich, ob und wieviel von dem Ganzen dass ich aktiv steuere durch Ambivalenz, Unsicherheit und ein mich anpassen an die Situation

K21: 04.11. 16:28 - Ist das präziser?

C21: 04.11. 16:30 - Wenn ich Sie richtig verstehe, dann ist die aktuelle Situation unbefriedigend, weil Sie gerne mehr arbeiten möchten und nicht wissen, ob das im aktuellen Job möglich ist. Und Sie ambivalent sind ob das aktuell der richtige Arbeitsort für Sie ist? K22: 04.11. 16:33 - Es ist irgendwie anders. Mir gefällt die Arbeit und der Ort. (auch wenn ich nach einem Jahr die Leute gut bis sehr gut kenne, und deren Eigenheiten zum Teil als suboptimal empfinde. Aber mich dünkt, das treffe ich in jedem Betrieb, da halt irgendwie menschlich) mir ist bewusst, dass ich nicht den Betrieb steuern kann. Ich frage mich aber, wieviel kann ich als Mitarbeiterin beeinflussen, um mehr Aufträge und auch die richtigen zu bekommen und inwieweit halte ich mich in der aktuellen Situation vielleicht auch zu stark zurück.

Analyse und Bearbeitung In Folge der Aufforderung der Coachin (C20) versucht die Klientin ihr Problem zu präzisieren (K19-K20), worauf sie den Turn mit einer (metakommunikativen) Rückfrage zur Verstehensabsicherung abschließt (K21). Die Coachin geht nicht explizit auf die Frage ein, sondern reagiert stattdessen mit einer Reformulierung der Problembeschreibung in Form einer Frage (C21). Dabei zeigt sich auf der thematischen Ebene eine Verschiebung vom in K19 thematisierten Sachverhalt meine Sicht der Dinge auf die reale aktuelle Situation am Arbeitsplatz (C21). Dass diese reformulierende Handlung nicht dem von der Klientin Gemeinten entspricht, zeigt ihre Ablehnung des Deutungsangebots und der darauffolgende Reformulierungsversuch (K22).

Die reformulierende Handlung in C21 erreicht das Ziel einer wechselseitigen Verstehensabsicherung an dieser Stelle nicht. Aus Sicht der Coachin liegt jedoch gewissermaßen ein Glücksfall vor, weil sie von der Klientin die explizite Rückmeldung erhält, dass noch kein gemeinsames Verständnis des Problems vorliegt. In einem Training können ausgehend von solchen authentischen Beispielen Varianten reformulierender Handlungen verfasst und diskutiert werden, wobei wiederum die medialen Bedingungen miteinbezogen werden müssen. Gerade weil der Turnwechsel bedeutend langsamer stattfindet als im mündlichen Gespräch, scheint im Chat ein hoher Grad an inhaltlich-thematischer Responsivität ${ }^{8}$ (Schwitalla 1979) zentral, um in der beschränkt vorhandenen Zeit zu einem gemeinsamen Verständnis zu gelangen. ${ }^{9}$ Anhand dieses Gesprächs kann auch gezeigt werden, dass reformulierende Handlungen dann zu einer Herausforderung werden, wenn die Erläuterungen der Klientin sowohl sprachlich als auch inhaltlich-strukturell schwer verständlich sind (vgl. K20). Im Training können auch alternative Sprechhandlungstypen (z. B. direkte Nachfragen zur Verstehensabsicherung) diskutiert und erprobt werden.

\subsection{Rollenzuweisungen}

Als Basisprinzip für jegliche Interaktion gilt das sequentielle Ordnungsprinzip von Paar-Sequenzen, den sogenannten ,adjacency pairs“ (Schegloff 2007). Dieses besagt, dass durch die Handlung eines ersten Paarteils (first pair part) eine bestimmte Folgehandlung (second pair part) erwartbar wird. Diese (bedingte) Erwartbarkeit der Folgehandlung wird auch als „konditionelle Relevanz“ bezeichnet. Klassische Adjazenz-Paare sind Gruss - Gegengruss, Frage - Antwort, Angebot - Annahme/Ablehnung. Dieses sequentielle Ordnungprinzip ist zugeich ein zentrales Mittel der Gesprächssteuerung und durch die institutionelle Einbettung des Gesprächs mehr (z. B. im Polizeiverhör) oder weniger stark (z. B. in Lehrgesprächen) vorgeprägt. Gerade im medizinischen Kontext (z.B. in Anamnesegesprä-

\footnotetext{
8 Mit den von Schwitalla (1979) definierten Graden an Responsivität (responsiv, teil-responsiv, non-responsiv) lassen sich Turnverknüpfungen genauer bestimmen. Responsivität ist dann gegeben, wenn auf der Handlungs- und Themaebene die Erwartungen von Sprecher A durch Sprecher B erfüllt werden. Teil-responsivität bedeutet, dass den Erwartungen nur teilweise, also nicht auf beiden Ebenen, entsprochen wird. Bei non-responsivem Verhalten werden die Erwartungen auf beiden Ebenen nicht erfüllt.

9 Die Analyse des weiteren Gesprächsverlaufs im ausgewählten Gespräch zeigt, dass bis zum Schluss die wechselseitige Verständigung über das Anliegen problematisch bleibt.
} 
chen), aber auch im Beratungskontext kommt etwa dem Aspekt des Fragens eine besondere Bedeutung zu. Durch das Fragen treibt der Coach das Gespräch voran und hat die Möglichkeit, den Handlungs- und Themenverlauf zu steuern. Gleichzeitig werden durch die Art des Fragens auch die interaktionalen Rollen im Gespräch beeinflusst, wie der folgende Ausschnitt exemplarisch zeigt:

C26: 11.11. 19:04 hast du früher mal gespendet? oder dich für eine organisation interessiert, dann aber aus zeitgründen die evaluation, die du angesprochen hast, nicht durchführen können?

K25: 11.11. 19:06 Nein ich habe noch nie regelmässig an eine organisation gespenndet.

K26: 11.11. 19:07 Ernsthaft evaluiert auch nicht.

C27: 11.11. 19:08 und würdest du in zukunft gerne regelmässig für jemanden spenden?

C28: 11.11. 19:08 du darfst ganz ehrlich antworten

K27: 11.11. 19:09 Ja, eigentlich schon.

C29: 11.11. 19:10 ok. denkst du, dass du dich unwohl fühlst bei spendeständen hängt damit zusammen, dass du eigentlich spenden möchtest, aber (noch) nicht tust?

C30: 11.11. 19:11 oder anders gefragt: denkst du, es könnte dein unwohlsein verringern, wenn du für eine organisation spenden würdest?

K28: 11.11. 19:11 Ja und Ja

C31: 11.11. 19:12 und grade fehlen dir aber noch so ein bisschen die kriterien, nach denen du eine geeignete organisation finden kannst, für die du gerne spenden würdest?

K29: 11.11. 19:13 Ja, das kann man so sagen. Weil ja eigentlich alle gutes tun.

Analyse und Bearbeitung Die Frage der Coachin (C26) enthält insgesamt drei thematisch unterscheidbare Sachverhalte, die in Form von einer Entscheidungsfrage thematisiert werden: Erfahrung mit Spenden, Interesse für eine Organisation, Nichtdurchführung der Evaluation (mit Angabe des Grundes: Zeitgründe). Die Klientin beantwortet die Fragen (K25, K26), ohne jedoch selber einen initiierenden Schritt anzuhängen. Es folgt eine weitere Entscheidungsfrage von Seiten der Coachin (C27), an die ein metakommunikativer Hinweis (C28) angeschlossen wird. Wiederum erfolgt von der Klientin lediglich ein reaktiver Schritt in Form einer kurzen Bejahung, die durch das Modalwort eigentlich jedoch eingeschränkt/abgeschwächt wird. Statt einer Nachfrage folgt in C29-C30 ein Deutungsangebot (Grund für das Unwohlsein) in Frageform. Die Klientin antwortet lediglich mit einem zweimaligen $J a$ (K28) und bleibt damit weiterhin beim rein reaktiven Interaktionsverhalten. Darauf reagiert die Coachin (C31) mit einem weiteren Deutungs- angebot in Form einer Entscheidungsfrage, das die Klientin annimmt und mit einer kurzen Begründung ergänzt (K29).

Im Kommunikationstraining kann anhand des Beispiels gezeigt werden, dass mit der Art des Fragens hier auch Rollenzuweisungen erfolgen und damit auch das Interaktionsverhalten der Klientin gesteuert wird. So wird ein eher passives Verhalten der Klientin durch Formen des geschlossenen Fragens tendenziell unterstützt. Während beim Erfragen von Fakten (z. B. C26) Entscheidungsfragen sinnvoll und effizient erscheinen, ist zu diskutieren, ob diese auch dort, wo es z. B. um Formen der Klärung geht (C29, C30, C31), zielführend sind.

\section{Fazit}

Ein gesprächsanalytisches Kommunikationstraining mit dem Ziel einer Anwendung in der Praxis liegt in einem Spannungsfeld zwischen wissenschaftlichen Ansprüchen einerseits und zeitlichen/ökonomischen Rahmenbedingungen andererseits. Es soll einerseits empirisch fundiert sein und sich damit von der häufig erfahrungsbasierten und subjektiven Ratgeberliteratur abgrenzen, andererseits muss das gesprächsanalytische Beschreibungsverfahren so angelegt sein, dass innerhalb von mehr oder weniger kurzer Zeit Erkenntnisse aus dem Material gewonnen werden können, die als Trainingsgegenstand für ein Zielpublikum aus fachfremden Bereichen fungieren und die gestellte Erwartung an eine Erweiterung der Gesprächskompetenz erfüllen können.

Mit der hier gewählten Beschreibungsebene der Gesprächssteuerung wurde versucht, eine im oben genannten Sinn operationalisierbare und zugleich für die Gesprächspraxis relevante Ebene zu beleuchten. Anhand der Chat-Auszüge konnten exemplarisch vier verschiedene Merkmale der Gesprächssteuerung (Gesprächsorganisation durch Sprecherwechsel und Orientierungshandlungen, Themensteuerung durch Reformulierungshandlungen sowie Handlungssteuerung durch Fragen) identifiziert werden, welche in ihrer Gestaltung einen Einfluss auf die Gesprächseffizienz haben und somit relevante Trainingsgegenstände darstellen.

Ein zentrales Element im CARM-Training ist die ,lineby-line"-Präsentation des Transkripts, die mit dem AudioFile synchronisiert wird. Die Anwendung einer solchen Präsentation wäre prinzipiell auch für das Medium des Chats denkbar, sofern nicht wie im vorliegenden Fall Chat-Mitschnitte, sondern Bildschirmverlaufsprotokolle verwendet würden. Der Vorteil solcher Verlaufsprotokolle ist, dass sämtliche auf dem Bildschirm ablaufenden Aktivitäten aufgezeichnet werden. Das ermöglicht einen tieferen und prozessnahen Einblick in die Produktion und sequentielle Abfolge von Turns. Der Nachteil ist jedoch das im Vergleich 
mit Chat-Mitschnitten aufwändigere Aufzeichnungs- und Transkriptionsverfahren.

Da man aus ökonomischen Gründen von einer relativ kurzen Zeitspanne zwischen dem Erhalt von Gesprächsmaterial und der Aufbereitung relevanter Erkenntnisse für ein zielgruppenorientiertes Training ausgehen muss, ist eine Analyse, die der Komplexität von Gesprächen gerecht wird, nicht möglich. Umso wichtiger ist, dass CoachingGespräche vermehrt zum Gegenstand detaillierter empirischer Analysen werden, da erst solche Arbeiten einen differenzierten Einblick in die einzelnen Aktivitäten, aber auch den Coaching-Prozess insgesamt sowie dessen institutionellen/organisationalen Kontext ermöglichen. ${ }^{10}$

Was die Gesprächssteuerung betrifft, so wäre genauer zu untersuchen, inwiefern mangelhafte oder nicht vorhandene Orientierungshandlungen zu Beginn des Gesprächs Auswirkungen auf den Gesprächsverlauf, den Coaching-Prozess und dessen Gelingen haben; so wäre genauer zu eruieren, welche Sprechhandlungstypen (z. B. Fragen) zu welchen reaktiven Handlungen der Klientenseite führen und welche Implikationen dies bzgl. Handlungsablauf und Beziehungskonstitution hat; so wäre genauer zu beschreiben, welche Formen und Funktionen von Reformulierungshandlungen im Chat-Coaching vorkommen und welche Bedeutung ihnen beikommt. Diese Fragen münden letztlich in die zentrale Frage nach dem Zusammenhang zwischen dem Ablauf und der Gestaltung von Gesprächsprozessen einerseits und deren Resultat andererseits. Damit wird nicht nur aus Sicht der Gesprächsanalyse, sondern auch aus Sicht der Coaching-Forschung das noch kaum bearbeitete Forschungsfeld der Wirkungs-Wirkfaktorenforschung betreten. $\mathrm{Zu}$ prüfen wäre hier u. a., ob die Gesprächsanalyse mit Methoden der Wirksamkeitsforschung (z. B. Wirkfaktorenanalyse) verknüpft werden kann, um auf die entscheidende Frage nach dem Verhältnis zwischen dem Was und dem Wie von Coachingprozessen Antworten zu finden.

Bei der Analyse wurde - in Orientierung an CARM - ein Workshop-Setting angenommen. Dabei wurde noch nicht spezifisch auf Fragen der didaktischen Umsetzung eingegangen. Gerade diese Fragen sind jedoch, nicht zuletzt in Hinblick auf das Medium des Chats, zentral. Da es sich um „getippte Dialoge“ (Dürscheid und Brommer 2009, S. 4) handelt, scheint es bspw. angebracht, dass die Teilnehmenden nicht medial mündliche, sondern schriftliche Varianten von Turns formulieren. Damit sind jedoch auch in didaktischer Hinsicht neue Ansätze gefragt, da weder die Gesprächsdidaktik noch die traditionelle Schreibdidaktik dem Gegenstand ganz gerecht werden können. Vielmehr öffnet

\footnotetext{
10 Neben den Untersuchungen generell zum Handlungsmuster Beraten (vgl. Schank 1979, 1981; Nothdurft et al. 1994) liegen mittlerweile auch empirische Studien zu Coaching-Gesprächen (Face to Face) vor (vgl. Schreyögg 2014; Graf 2015a, 2015b).
}

sich auch hier ein neues Feld des dialogischen Schreibens in institutionellen Kontexten, für das noch adäquate didaktische Ansätze zu entwickeln sind.

Open Access Dieser Artikel wird unter der Creative Commons Namensnennung 4.0 International Lizenz (http://creativecommons.org/ licenses/by/4.0/deed.de) veröffentlicht, welche die Nutzung, Vervielfältigung, Bearbeitung, Verbreitung und Wiedergabe in jeglichem Medium und Format erlaubt, sofern Sie den/die ursprünglichen Autor(en) und die Quelle ordnungsgemäß nennen, einen Link zur Creative Commons Lizenz beifügen und angeben, ob Änderungen vorgenommen wurden.

\section{Literatur}

Antaki, C. (2011). Six kinds of applied conversation analysis. In C. Antaki (Hrsg.), Applied conversation analysis: intervention and change in institutional talk (S. 1-14). Basingstoke: Palgrave Macmillan.

Von Arx, E., \& Rüsch, S. (2016). „C-Cube“ - Wirkfaktorenanalyse im Einzelchat-Coaching. Entwicklung eines Rating-Instruments zur Analyse von Coaching-Interventionen und Beziehungsverhalten von Coach und Klient. Unveröffentlichte Masterarbeit am Departement Angewandte Psychologie, ZHAW

Becker-Mrotzek, M. (Hrsg.). (2009). Mündliche Kommunikation und Gesprächsdidaktik (S. 66-83). Baltmannsweiler: Schneider.

Becker-Mrotzek, M., \& Brünner, G. (2006). Gesprächsanalyse und Gesprächsführung. Eine Unterrichtsreihe für die Sekundarstufe II. Radolfzell: Verlag für Gesprächsforschung. Verfügbar unter http://www.verlag-gespraechsforschung.de

Beisswenger, M. (2007). Sprachhandlungskoordination in der ChatKommunikation. Berlin: De Gruyter.

Brinker, K., \& Sager, S.F. (2010). Linguistische Gesprächsanalyse. Eine Einführung. Berlin: Schmidt.

Bührig, K. (1996). Reformulierende Handlungen. Zur Analyse sprachlicher Adaptierungsprozesse in institutioneller Kommunikation. Tübingen: Narr.

Deppermann, A. (2008). Gespräche analysieren. Eine Einführung (4. Aufl.). Wiesbaden: VS.

Dürscheid, C., \& Brommer, S. (2009). Getippte Dialoge in neuen Medien. Sprachkritische Aspekte und linguistische Analysen. Linguistik Online, 37(1), 1-20. Verfügbar unter https://bop.unibe.ch/ linguistik-online/article/view/255/344.

Goffman, E. (1974). Das Individuum im öffentlichen Austausch. Mikrostudien zur öffentlichen Ordnung. Frankfurt am Main: Suhrkamp.

Graf, E. (2015b). Linguistische Evaluation eines Coaching-Prozesses - Die Ko-Konstruktion des Anliegens durch Coach und Klientin. In H. Geissler \& R. Wegener (Hrsg.), Bewertung von CoachingProzessen (S. 211-229). Heidelberg: Springer.

Graf, E.-M. (2015a). Kommunikative Basisaktivitäten im CoahingGespräch. Ein linguistischer Beitrag zur Coaching-Prozessforschung. Coaching Theorie \&amp; Praxis, 1(1), 5-14. doi:10. 1365/s40896-015-0001-x.

Harren, I. (2015). Fachliche Inhalte sprachlich ausdrücken lernen. Sprachliche Hürden und interaktive Vermittlungsverfahren im naturwissenschaftlichen Unterrichtsgespräch in der Mittel- und Oberstufe. Mannheim: Verlag für Gesprächsforschung. Verfügbar unter http://www.verlag-gespraechsforschung.de

Hartung, M. (2004). Wie lässt sich Gesprächskompetenz wirksam und nachhaltig vermitteln? Ein Erfahrungsbericht aus der Praxis. In M. Becker-Mrotzek \& G. Brünner (Hrsg.), Analyse und Vermittlung von Gesprächskompetenz (S. 47-66). Frankfurt am Main: Lang.

Henne, H. (1980). Probleme einer historischen Gesprächsanalyse. Zur Rekonstruktion gesprochener Sprache im 18. Jahrhundert. In H. 
Sitta (Hrsg.), Ansätze zu einer pragmatischen Sprachgeschichte. Zürcher Kolloquium 1978 (S. 89-102). Tübingen: Niemeyer.

Hepburn, A., Wilkinson, S., \& Butler, C.W. (2014). Intervening with conversation analysis in telephone helpline services: strategies to improve effectiveness. Research on Language and Social Interaction, 47(3), 239-254.

Herring, S. C. (2001). Computer-mediated discourse. In D. Schiffrin, D. Tannen \& H.E. Hamilton (Hrsg.), The handbook of discourse analysis (S. 612-634). Oxford: Blackwell.

Hess-Lüttich, E.W.B. (1981). Soziale Interaktion und literarischer Dialog. Berlin: Schmidt.

Hess-Lüttich, E. W. B., \& Wilde, E. (2003). Der Chat als Text und/oder als Dialogsorte? Linguistik Online, 13(1), 161-180. Verfügbar unter https://bop.unibe.ch/linguistik-online/article/view/876/1527.

Kilian, J. (2005). Historische Dialogforschung. Eine Einführung. Tübingen: Niemeyer.

Koch, P., \& Oesterreicher, W. (1985). Sprache der Nähe - Sprache der Distanz. Mündlichkeit und Schriftlichkeit im Spannungsfeld von Sprachtheorie und Sprachgeschichte. Romanistisches Jahrbuch, $36,15-43$.

Lalouschek, J. (2002). Ärztliche Gesprächsausbildung: Eine diskursanalytische Studie zu Formen des ärztlichen Gesprächs. Mannheim: Verlag für Gesprächsforschung. Verfügbar unter http:// www.verlag-gespraechsforschung.de

Lalouschek, J. (2004). Kommunikatives Selbst-Coaching im beruflichen Alltag. Ein sprachwissenschaftliches Trainingskonzept am Beispiel der klinischen Gesprächsführung. In M. Becker-Mrotzek \& G. Brünner (Hrsg.), Analyse und Vermittlung von Gesprächskompetenz (S. 137-158). Frankfurt am Main: Lang.

Lalouschek, J. (2005). Inszenierte Medizin: Ärztliche Kommunikation, Gesundheitsinformation und das Sprechen über Krankheit in Medizinsendungen und Talkshows. Mannheim: Verlag für Gesprächsforschung. Verfügbar unter http://www.verlaggespraechsforschung.de

Meer, D. (2007). ,ich wollte ja eigentlich mittagessen.“ - Zur Notwendigkeit und den Möglichkeiten der Didaktisierung gesprächsanalytischer Daten für Fortbildungszwecke. Gesprächsforschung, 8, $117-159$

Meer, D., \& Spiegel, C. (Hrsg.). (2009). Erfahrungen mit gesprächsanalytisch fundierten Fortbildungskonzepten. Mannheim: Verlag für Gesprächsforschung. Verfügbar unter http://www.verlaggespraechsforschung.de

Nothdurft, W., Reitmeier, U., \& Schröder, P. (1994). Beratungsgespräche. Analyse asymmetrischer Dialoge. Tübingen: Narr.

Nowak, P. (2010). Eine Systematik der Arzt-Patient-Interaktion. Systemtheoretische Grundlagen, qualitative Synthesemethodik und diskursanalytische Ergebnisse zum sprachlichen Handeln von Ärztinnen und Ärzten. Frankfurt am Main: Lang.

Ribbers, A., \& Waringa, A. (2015). E-Coaching. Theory and practice for a new online approach to coaching. London: Routledge.
Sacks, H., Schegloff, E., \& Jefferson, G. (1974). A simpliest systematics for the organization of Turn-Taking in conversation. Language, 50, 696-735.

Schank, G. (1979). Zum Ablaufmuster von Kurzberatungen - Beschreibung einer Gesprächsstruktur. In J. Dittmann (Hrsg.), Arbeiten zur Konversationsanalyse (S. 176-197). Berlin: De Gruyter.

Schank, G. (1981). Untersuchungen zum Ablauf natürlicher Dialoge. München: Hueber.

Schegloff, E. (2007). Sequence organization in interaction. A primer in conversation analysis I. Cambridge: Cambridge University Press.

Schreyögg, B. (2014). Emotionen im Coaching: Kommunikative Muster der Beratungsinteraktion. Wiesbaden: Springer. doi:10.1007/ 978-3-658-07994-9.

Schulz von Thun, F. (2014). Miteinander reden. Reinbek bei Hamburg: Rowohlt.

Schwitalla, J. (1979). Nonresponsive Antworten. Deutsche Sprache, 7, 193-211.

Seidlitz, H., \& Theiss, D. (2008). Ressourcenorientierte Telefonberatung: Ein Lehr- und Lernbuch. Basel: SolArgent Media AG.

Spiegel, C. (2006). Unterricht als Interaktion: Gesprächsanalytische Studien zum kommunikativen Spannungsfeld zwischen Lehrern, Schülern und Institution. Radolfzell: Verlag für Gesprächsforschung. Verfügbar unter http://www.verlag-gespraechsforschung. de

Stokoe, E. (2011). Simulated interaction and communication skills training: the „Conversation-Analytic Role-Play Method“. In C. Antaki (Hrsg.), Applied conversation analysis: intervention and change in institutional talk (S. 119-139). Basingstoke: Palgrave Macmillan.

Stokoe, E. (2013). The (in)authenticity of simulated talk: comparing role-played and actual interaction and the implications for communication training. Research on Language and Social Interaction, 46(2), 165-185.

Stokoe, E. (2014). The conversation analytic role-play method (CARM): a method for training communication skills as an alternative to simulated role-play. Research on Language and Social Interaction, $47(3), 255-265$.

Storrer, A. (2001). Getippte Gespräche oder dialogische Texte? Zur kommunikationstheoretischen Einordnung der Chat-Kommunikation. In A. Lehr, M. Kammerer, K.-P. Konerding, A. Storrer, C. Thimm \& W. Wolski (Hrsg.), Sprache im Alltag. Beiträge zu neuen Perspektiven in der Linguistik (S. 439-465). Berlin: de Gruyter.

Thaler, V. (2003). Chat-Kommunikation im Spannungsfeld zwischen Oralität und Literalität. Berlin: VWF.

Tiittula, L. (2001). Formen der Gesprächssteuerung. In K. Brinker, G. Antos, W. Heinemann \& S.F. Sager (Hrsg.), Text- und Gesprächslinguistik: Ein internationales Handbuch zeitgenössischer Forschung 2. Halbband. (S. 1361-1374). Berlin: De Gruyter. 